\title{
Optimal Allocation of TV White Spaces to White Space Devices using Genetic Algorithm and Fuzzy Rule based Technique
}

\author{
Adigwe Wilfred \\ Member, Nigeria Computer Society (NCS), \\ Member, Computer Professionals Registration \\ Council of Nigeria (CPN) \\ Department of Computer Science \\ Delta State Polytechnic Ozoro, Delta State Nigeria
}

\author{
Raphael Obikwelu Okonkwo \\ Member, Nigeria Computer Society (NCS), \\ Member, Computer Professionals Registration \\ Council of Nigeria (CPN) \\ Department of Computer Science \\ Nnamdi Azikiwe University, Awka, Nigeria
}

\begin{abstract}
TV Whitespace (TVWS) refers to the unoccupied portions of spectrum in the VHF/UHF terrestrial television frequency bands. When developing a TV White Space (TVWS) system with the available TV spectrum after digital switchover, channel allocation for TVWS devices to avoid interference becomes one of the most challenging problems. Analysis has shown that there exist large quantities of these TVWS in many locations in Nigeria especially with the ongoing digital switch over. Having a proper mechanism for the allocation of these TVWS to devices known as white space devices (WSD) have been a serious concern. Thus, this paper proposes a hybrid model of fuzzy rule based technique and genetic algorithm for optimal allocation of the TVWS. The result shows that the available TVWS were maximally allocation to WSD on a real time basis. The implementation of this model will bring an alternative source for most smart devices that have been competing for the scarce spectrum as raised by the communication industries. These devices can be deployed to these unutilized spectra at low or no cost thereby freeing most of the spectrum bands.
\end{abstract}

\section{Keywords}

TV White Space (TVWS); VHF/UHF; Spectrum; Genetic Algorithm (GA), Fuzzy, White Space Devices (WSD)

\section{INTRODUCTION}

Following the roll out of commercial mobile communication networks in many parts of the world, the radio frequency is becoming more valuable than ever. Individuals, businesses and governments rely on this natural resource for communication. The growth of Internet as a standard communication platform fueled the emergence of new services like wireless Internet access. As more devices are competing for wireless access, the available radio spectrum is becoming congested. The reserve radio resource pool is also depleting. Hence, in the future new allocations will inevitably become impossible, risking the growth of the whole wireless ecosystem come to a halt. On the contrary, studies (from various measurement campaigns) have shown that the current spectrum shortage is created by the tight regulatory schemes, introduced long ago to protect exclusive spectrum users [1].

Television broadcasting services operate in the Very High Frequency (VHF) and Ultra High Frequency (UHF) portions of the radio frequency, on a licensed basis [2]. Regulators prohibit the use of these bands for unlicensed devices. Most regulators, however, are necessitating the adoption of digital transmission by all $\mathrm{TV}$ stations that were using analogue transmission. The Digital Switchover (DSO) is ongoing in Nigeria, though countries like US completed hers since June 2009, while UK and Finland also completed theirs. There are so many benefits offered by the DSO, majorly it provides great opportunity to use the licensed TV bands for unlicensed wireless devices when licensed users do not need them. There are suitable geographical locations which could not be accessed by some channels without interfering with their adjacent/co-channels, but still these channels could be used by low power devices without disturbing similar primary users. These secondary devices are also known as White Space Devices (WSDs). Analysis in [3] shows there is abundant existence of TV spaces but needed proper allocation mechanism for the benefits of the WSDs.

\section{RELATED WORKS}

Channel allocation has been a serious challenge in the communication industries, a lot of research have been made in this regard with different solutions by different authors. Some of the methods are reviewed in this section.

Gawde et. al., [4] proposed spectrum assignment scheme based on Genetic Algorithm for Cognitive Radio Receiver. Their work focuses on the performance of spectrum allocation technique, based on popular meta-heuristics Genetics Algorithm. The performance analysis of their technique using Matlab shows that they achieved mean fitness of 9.41. Their also provides fittest channels to the cognitive user on the basis of four priority parameters (genes) viz frequency, power, BER and modulation.

Nishant [5] on did an investigation on the dynamic channel assignment (DCA) in mobile communications systems using genetic algorithm (GA) in his research. Two new strategies using GA were proposed. In the first one, the channels previously assigned are kept locked during the call holding time (GAL). In the second one, the calls can be switched to different channels during the connection time (GAS). His work reviewed the available schemes for channel allocation.

Clemens and Rose [6] considered intelligent power allocation strategies in an unlicensed spectrum. They consider power allocation strategies for radios operating in unlicensed bands. Since each radio's power allocation is a source of interference for other radios sharing the same spectrum, in their work they develop "intelligent" power allocation strategies that not only greedily optimize performance, but also tend toward socially optimal solutions. They modeled Radio interaction as a two- 
player repeated gamewhere players develop "reputations" based on previous actions.

Jayaraj et. al., [7] made an analysis of Genetic Algorithm and Tabu search algorithm for channel optimization in cognitive adhoc networks. Their work compared the techniques used to optimize the secondary user's performance in channel allocation. Their results show that performance evaluation of Single Channel combined TDMA/CSMA system shows that the two systems can operate together. With a total traffic load of 1 Erlang (the maximum the channel can support) the total throughput was close to 0.55 Erlangs, showing only $55 \%$ of the channel capacity is being used [8]

Yongcheng et. al., [9] proposed spectrum allocation of cognitive radio based on autonomy evolutionary algorithm. Their analyzed the application of bio-inspired intelligent algorithm in cognitive radio network spectrum allocation, and based on graph theory model of spectrum allocation, they proposed a spectrum allocation algorithm based on autonomously evolutionary scheme. Three objective functions were employed: Max-Min-Reward, Max-Sum-Reward and Max-Proportional-Fair to evaluate the proposed algorithm capacity. Their simulation result revealed that the proposed method can make the system user to obtain better network benefits and better embody the fairness between cognitive users. In the process of allocation, the proposed method was not restricted by user scale and the number of spectrums.

Ogude and Onyejegbu [10] introduced a framework for the development of an optimized solution for quality of service delivery using fuzzy logic and genetic algorithm. In their work they expounded the fact that Quality of Service (QoS) plays a major role in selecting web services in terms of qualitative measure. They also reviewed existing selection systems and their designs and then presented a fuzzy and genetic algorithms based system to provide optimality in web services selection.

\section{MOTIVATION}

The main contributions of this paper are the following:

1. Developing a hybrid model that took the advantages of genetic algorithm and fuzzy rule based technique for optimal allocation of TV white spaces to white space devices was achieved. The method utilizes existing methods in the literature, namely an optimized service selection system using fuzzy logic and genetic algorithm [10]. It is found that there are poor allocation methods of the TV white spaces.

2. Motivated by poor allocation of TV white spaces to the requesting white space devices. It is observed that at least $80 \%$ UHF/VHF TV band channels are underutilized from the analysis done in the work, modeling TV white spaces availability prediction for white space devices utilization in delta north zone of delta state Nigeria [3]. The effect of improper allocation of the available TV white spaces tantamount to underutilization of the channels in the areas where there is a availability. The optimal mechanism(s) for the use of TV white spaces in Nigeria especially with the ongoing digital switch over is of a great concern in this work.

\section{REASONS FOR USING THE HYBRID TECHNIQUES}

Fuzzy systems are rule based systems (knowledge based systems). The rule base of a fuzzy system is composed of fuzzy IF-THEN rules that are similar to the rules used by humans in their reasoning. The challenges this work would have faced if only the fuzzy rule based technique alone was used are as follows:

i. It is hard to obtain optimal fuzzy set due to the number of generated rules as the number of system variables are increased,

ii. The fuzzy set doesn't have learning capability, it depends on a pre-defined set of rules which depends on the numbers of inputs that are to be processed, and

iii. The problem of defining the rule base:

a. Redundant rules: whose actions are covered by other rules.

b. Wrong rules: badly defined, thereby upsetting the system performance, and

c. Conflicting rules: worsens the system performance when co-existing with other rules in the RB (rule base).

The above challenges made the system performance less efficient and effective due to fact that the TV white space allocation results to.

i. Misdetection of the TV white space.

ii. Increase in the interference between the white space devices and the primary users and

iii. Inability to determine the signal strength leading to poor quality of service.

Thus, this work is saddled with the responsibility of overcoming the aforementioned challenges and this has been the main driving force behind the design of a more efficient and effective system. The proposed hybrid technique model of fuzzy rule based system with genetic algorithm was employed to avoid premature convergence problem.

\section{Genetic Algorithm}

The genetic algorithm (GA) [11] is an optimization and search technique based on the principles of genetics and natural selection. A GA allows a population composed of many individuals to evolve under specified selection rules to a state that maximizes the "fitness" (i.e., minimizes the cost function). This optimization method was adopted in this work because of its numerous advantages and has the ability to adapt to the radio environment. Genetic algorithm is more popular for their rapidity to cover a large space of possible configuration, and thus find the most suitable solution. The genetic algorithm is a method for solving both constrained and unconstrained optimization problems, basically which is based on natural selection, the process that drives biological evolution. The genetic algorithm repeatedly renovates a population of individual solutions. Genetic algorithm can be applied to solve a several optimization problems that are not well suited for standard optimization algorithms, including various problems in which the objective function is discontinuous, non-differentiable, or highly nonlinear.

The genetic algorithms (G.A.) are typically characterized by the following aspects:

- The G.A. work with the base in the code of the variables group (artificial genetic strings) and not with the variables in themselves. 
- The G.A. work with a set of potential solutions (population) instead of trying to improve a single solution.

- The G.A. do not use information obtained directly from the object function, of its derivatives, or of any other auxiliary knowledge of the same one.

- The G.A. applies probabilistic transition rules, not deterministic rules.

The genetic algorithm process is quite simple; it only involves a copy string, partial string exchanges or a string mutation, all these in random form.

\section{PROPOSED SYSTEM DESIGN}

The proposed architecture for the optimal allocation of TV white spaces to white space devices is remodeled to meet the user's request. The composition of QoS properties is done with the help of fuzzy rule and fitness function based selection. The knowledge base is the most essential module of the fuzzy rule based systems. It contains and stores all data, information, rules and constraints used by fuzzy inference engine for solving difficult problems or tasks. The rules in our knowledge base were carefully chosen in conformity with the problems will intend solving. This is shown in figure 1 . The proposed system architecture for the optimal allocation of TV white spaces procedures are as follows:

i. White spaces devices make a vague request to the fuzzy white space availability; this queries the geo-location database for channel availability.

ii. Fuzzy white space selector analyze the given request by passing it to fuzzy inference engine

iii. Fuzzy Inference engine calls the knowledge base and evaluate the criteria's listed in the inference rule for optimal quality of service (QoS).

iv. Genetic Algorithms (GA) then optimizes the fuzzy system parameters.

v. Finally, there is optimization of composition unit works based on Genetic Algorithms. Fuzzy rules, which are created based on user request together with user constraints, constitute one fitness function.

vi. GA parameters are defined for the system. QoS criteria's related to different compositions are evaluated and an optimized solution is selected in accordance with user request and convenience.

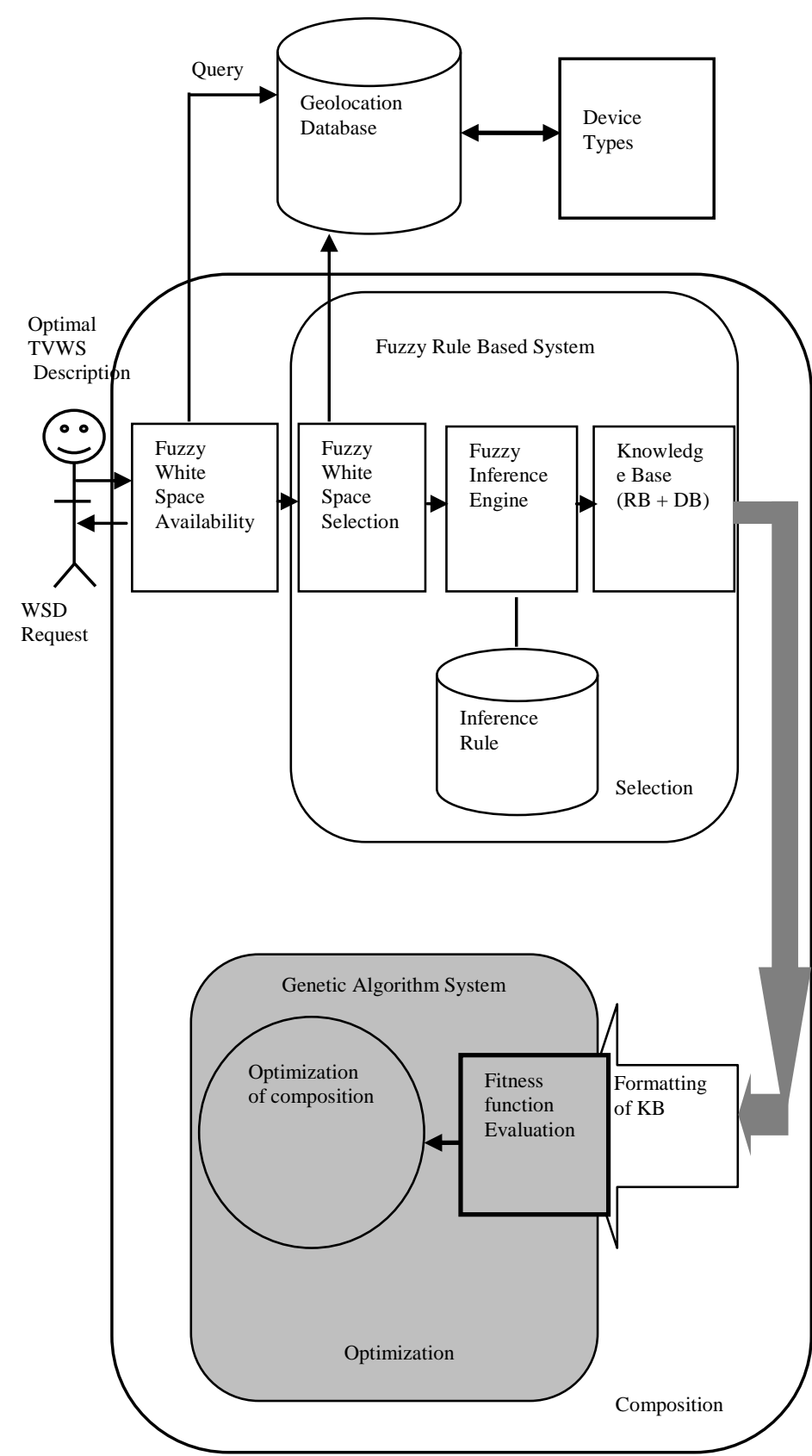

Fig 1: Proposed System Model 


\section{Rule 1}

$$
\text { IF channel Available THEN Weight }=0.5
$$

Rule 2

$$
\text { IF channel unavailable THEN Weight }=0.1
$$

Rule 3

Rule 4

$$
\text { IF channel Available AND Free THEN Weight }=0.8
$$

IF channel Available AND Not free THEN Weight = 0.4

Rule 5

IF channel Available AND channel free AND signal strength $=$ LOW THEN Weight $=0.3$

Rule 6

IF channel Available AND channel free AND signal strength $=$ HIGH THEN Weight $=0.7$

Rule 7

IF channel Available AND channel free AND channel unoccupied AND signal strength HIGH THEN Weight $=0.9$

Rule 8

IF channel Available AND channel free AND channel unoccupied AND signal strength LOW THEN Weight $=0.99$

Rule 9

IF channel Available AND channel free AND channel occupied AND signal strength HIGH THEN Weight $=0.6$

Rule 10

IF channel Available AND channel NOT occupied AND signal strength HIGH AND channel neighbor to NOT free channel THEN Weight $=0.2$

\section{Fig 2: Knowledge Base}

\subsection{Layout of Proposed System Design}

User Interface: The white space device should be able to sense the spectrum by communicating with the Geo-location Database (GDB). It is an automated process since the user must first indicate the use of a whitespace. This is where the geo-location database serves a great deal in this work. The geo-location database contain information about each spectrum, such as available whitespace at any point in time, the status of the primary user for that spectrum both licensed and unlicensed spectrums, the quality of services parameters just to mention but a few. This geolocation database locates appropriate whitespaces using the requirements and selection criteria.

i. GDB contains information about the white spaces, it also register and keeps device information ii. GDB provide directory for storing information about the White Space Devices (WSD) in that location, the information include: Device name, Device ID, specification, coverage radius, device status (ON, OFF or Broadcasting)

iii. GDB provide directory for White Space Agents

iv. It provides format for communication between devices.

White Space Availability: Checks and communicate the frequencies available in the considered location while monitoring interference.

Device Type: Locate and describe the type of device that is communicating in any given time and its characteristics.

Fuzzy Inference Engine (FIE): This is a very important component of the proposed system as it does the reasoning/or make decision using the rules stored in the knowledge base (KB). It is the brain of the system. It does this task by

i. Acting like a search engine examining the Knowledge Base for information that matches the user's query (question).

ii. It uses the query to search the Knowledge Base and then provides an answer or some advice to the user.

iii. It also utilizes the contents of Knowledge Base in conjunction with the data given by the user in order to achieve a conclusion.

Fuzzy Inference Rules (FIRs): The inference rules which is based on IF-THEN ELSE were carefully chosen in regards to the problem we intend solving and interference avoidance. The fuzzy inference rule technique is used to:

i. Calculate numerical results of the linguistic rules based on the system input values. The numerical results from this calculation are called fuzzy outputs. Also this is where all generated rules are evaluated.

Knowledge Base (KB): This is the most essential module of the fuzzy systems. It is referred to as the heart of the system. It contains and stores all data, information, rules and constraints used by fuzzy inference engine for solving difficult problems or tasks. In the proposed system, the knowledge base comprised of two components:

i. Data Base (DB), containing the definitions of the scaling functions of the variables and the membership functions of the fuzzy sets associated with the linguistic labels, and

ii. Rule Base (RB), constituted by the collection of fuzzy rules. A collection of rules used in the knowledge base of a fuzzy system. It has an IFAND/OR-THEN structure.

The performance of a fuzzy system depends largely on the control (IF-THEN rules) structure and selection of the Knowledge Base. Optimization of the Knowledge Base is critical to the performance of the fuzzy systems. GA provides such a method to optimize the Fuzzy System parameters.

GA Optimization Process: the GA is used for improving and designing the fuzzy system such as optimizing the system variables for an optimized solution 


\section{PROPOSED TV WHITE SPACE OPTIMIZATION MODEL}

Genetic algorithms evaluate the target function to be optimized at some randomly selected points of the definition domain. Taking this information into account, a new set of points (a new population) is generated. Gradually the points in the population approach local maxima and minima of the function. Adopting the genetic algorithm proposed by Rojas (1996), we can optimize channel selection for the WSDs. More so he affirmed that genetic algorithms are stochastic search methods managing a population of simultaneous search positions. In this dissertation, the three essential elements of conventional Genetic Algorithm (GA) proposed by Rojas were employed which comprises of:

- Selection

- Cross Over/ Recombination

- Mutation/Value Flipping

Therefore the probability $\boldsymbol{P}(\mathbf{G A})$ of our Genetic Algorithm (GA) being correct is given by the probability of the selection method $\boldsymbol{P}(\mathbf{S})$ and the probability of the Cross Over method $\boldsymbol{P}(\mathbf{C})$ and the probability of the mutation method $\boldsymbol{P}(\mathbf{M})$ all being correct. This assertion can be stated mathematically as:

$\mathrm{P}(\mathrm{GA})=\mathrm{P}(\mathrm{H})+\mathrm{W}+\mathrm{W}^{\prime}$

Given that the binary chromosome where the genes are a combination of binary bits 0 or 1 is adopted in this dissertation.

In our algorithm, the population is made up of a set of $N$ binary strings of length, $\boldsymbol{l}$ at a time $\boldsymbol{t}$. We call a string of length $l$ which contains one (1) of the three symbols 0,1 or * in each position a bit pattern or schema, where * is either a 1 or $a 0$.

A further look at equation 6.1, one can deduce that the probability $\mathrm{P}(\mathrm{GA})$ of our Genetic Algorithm producing the required bit pattern is equivalent to the probability of the child string containing the required bit pattern after mutation. This translates equation 6.1 to:

$\mathrm{P}(\mathrm{GA})=\mathrm{W}^{\prime}$

The number of strings in a population in a given generation $t$ which contains the bit pattern $\boldsymbol{H}$ is given by $\boldsymbol{o}(\boldsymbol{H}, \boldsymbol{t})$.

The diameter $\boldsymbol{d}(\boldsymbol{H})$ of a bit pattern $\boldsymbol{H}$, is defined as the length of the pattern's shortest substring that still contains all fixed bits in the pattern

The function $\boldsymbol{f}$, which is defined over all strings of length $\boldsymbol{l}$, is called the fitness of the string.

As we know, two parents' strings from the current population are always selected for the creation of a new string.

The probability that a parent string $H_{j}$ will be selected from $N$ strings $H_{1}, H_{2}, \ldots, H_{N}$ is given by

$$
P\left(H_{j}\right)=\frac{f\left(H_{j}\right)}{\sum_{\mathrm{i}=1}^{N} f\left(H_{i}\right)}
$$

This means that strings with greater fitness are more likely to be selected than strings with lesser fitness.
Where

P (GA) is the probability of the Genetic Algorithm

$\mathbf{P}(\mathbf{H})$ is the probability of selection of possible channels

$\mathbf{W}$ is the probability of crossover

$\mathbf{W}^{\prime}$ This is the probability of Mutation

$\mathbf{H}$ stands for possible available channels in the location under consideration.

$\mathbf{H}=$ (i) Channels usable immediately

(ii) Channels that is likely to be used

(iii) Channels that cannot be used (occupied)

Our $\mathbf{W}$ will be the probability of selecting (ii) above and also probability of selecting channels that cross over that is channels that were previously free but now occupied.

$\mathrm{W}^{\prime}$ stands for the proposed system ability to create channels within existing channels

$\boldsymbol{o}(\boldsymbol{H}, \boldsymbol{t})$ stands for the population of likely usable channels which comprises of free channels and cross over channels.

$\boldsymbol{o}$ stands for the population of those likely usable channels

$\boldsymbol{d}(\boldsymbol{H})$ This represent the conditions in the inference rule that can be used

$f$ stands for the channels that are fit to be considered

The average Fitness $\left(f_{\mu}\right)$ of all strings in the population is given by

$$
f_{\mu}=\frac{1}{N} \sum_{i=1}^{N} f\left(H_{i}\right)
$$

We now rewrite equ 6.4 as

$$
P\left(H_{j}\right)=\frac{f\left(H_{j}\right)}{N f_{\mu}}
$$

Therefore the probability that a parent string $\mathrm{H}_{\mathrm{j}}$ will be selected for regeneration is given by equation 3.28 above.

We go further to calculate the probability that a schema $\mathrm{H}$ will be passed on to a child string as follows:

\section{Step 1- Selection:}

The probability $\mathbf{P}$ that a string is selected which contains the bit pattern $\mathrm{H}$ is:

$$
P=\frac{f\left(H_{1}\right)}{N f_{\mu}}+\frac{f\left(H_{2}\right)}{N f_{\mu}}+\cdots \cdots+\frac{f\left(H_{k}\right)}{N f_{\mu}}
$$

Where $H_{l}, H_{2}, \ldots$.., $H_{k}$ represents all strings of the generation which contain the bit pattern $H$. if there are no such strings, the $P=0$.

The fitness $f(H)$ of the bit pattern $H$ in the generation $t$ is defined as 


$$
f(H)=\frac{f\left(H_{l}\right)+f\left(H_{2}\right)+\cdots \cdots+f\left(H_{k}\right)}{o(H, t)}
$$

When we substitute $f(H)$ in equ $3.30 \mathbf{P}$ will now become

$$
P=\frac{o(H, t) f(H)}{N f_{\mu}}
$$

Furthermore, the probability $P_{A}$ that two strings which contain pattern $H$ are selected as parent is given by:

$$
P_{A}=\left(\frac{o(H, t) f(H)}{N f_{\mu}}\right)^{2}
$$

Therefore, the probability $P_{B}$ that from two selected strings only one contains the pattern $\mathrm{H}$ is:

$$
P_{B}=2 \frac{o(H, t) f(H)}{N f_{\mu}}\left(1-\frac{o(H, t) f(H)}{N f_{\mu}}\right.
$$

\section{Step 2- Crossover:}

For the crossover of two strings a cut-off point is selected between position 1 and $l-1$ before crossover is executed.

The probability $\boldsymbol{W}$ that a schema $\boldsymbol{H}$ is transmitted to the new string depends on two cases:

Case 1: If both parent strings contain $\boldsymbol{H}$, then they pass on this substring to the new string.

Case 2: If only one of the strings contain $\mathrm{H}$, then the schema is inherited at most half the time

From the foregoing, it can be concluded that the probability $\boldsymbol{W}$ of an offspring inheriting a character trait/ substring from the parents is greater than or equal to by the probability of both or either parents selected for regeneration having the character trait and the probability that the character trait is not destroyed during crossover.

Given that the probability $P_{D}$ of the substring $\mathrm{H}$, being destroyed during crossover is:

$\mathrm{P}_{\mathrm{D}}=(d(H)-1) /(l-1)$

This implies that:

$$
W \geq=\left(\frac{o(H, t) f(H)}{N f_{\mu}}\right)^{2}+\frac{2}{2} \frac{o(H, t) f(H)}{N f_{\mu}}\left(1-\frac{o(H, t) f(H)}{N f_{\mu}}\right)\left(1-\frac{d(H)-1}{l-1}\right)
$$

Simplifying further we have:

$$
W \geq=\frac{o(H, t) f(H)}{N f_{\mu}} \quad\left(1-\frac{d(H)-1}{l-1}\left(1-\frac{o(H, t) f(H)}{N f_{\mu}}\right)\right)
$$

\section{Step 3 - Mutation:}

When two strings are recombined, the information contained in them is copied bit by bit to the child string. A mutation produces a bit flip with the probability $P$. This implies that a schema $H$ with $b(H)$ fixed bits will be preserved after copying with the probability $(1-p)^{b(H)}$. If mutation occurs, then the probability $W$, of the schema $H$ being passed on to a child string changes to $\mathrm{W}^{\prime}$ where

$$
W^{\prime} \geq=\frac{o(H, t) f(H)}{N f_{\mu}}\left(1 \frac{d(H)-}{{ }^{1}-1}\left(1 \frac{o(H, t)}{f(H) J f_{\mu}}\right)\right)_{P)^{b(H)}}^{1-}
$$

If in each generation $N$ new strings are produced, the expected value of the number of strings which contain $H$ in the generation $t+1$ is NW':

$$
=(o(H, t+1)) \geq \frac{o(H, t) f(H}{f_{\mu}}\left(1-\frac{d(H)-1}{l-1}\left(1-\frac{o(H, t) f(H)}{N f_{\mu}}\right)\right)(1-P)^{b(H)}
$$

This means that the number of the child string containing the schema $H$ after mutation is greater than or equal to the probability of the schema $H$ being passed on to the child string after mutation.

Now, we substitute the value of $\mathrm{W}^{\prime}$ into equation 6.15:

Recall equation 6.2 is:

$\mathrm{P}(\mathrm{GA})=\mathrm{W}^{\prime}$

Therefore, substituting equation 6.14 into equation 6.2 we have:

$$
P(G A) \geq=\frac{o(H, t) f(H)}{N f_{\mu}}\left(1-\frac{d(H)-1}{l-1}\left(1-\frac{o(H, t) f(H)}{N f_{\mu}}\right)\right)(1-P)^{b(H)}
$$


Table 1: Object Description/ Responsibility in the proposed System

\begin{tabular}{|c|c|c|}
\hline $\mathbf{S} / \mathbf{N}$ & Object & Description \& Responsibility \\
\hline 1. & TV White Space & $\begin{array}{l}\text { TV channels freed up when a } \\
\text { state transitions from Analog } \\
\text { TV (ATV) to Digital TV } \\
\text { (DTV) }\end{array}$ \\
\hline 2. & TVBD & $\begin{array}{l}\text { TV Band Device is a low } \\
\text { power transmitter that operates } \\
\text { in an unoccupied TV channel } \\
\text { in the range of channels } 2-51 \text {, } \\
\text { excluding channels } 3-4 \text { and } 37\end{array}$ \\
\hline 3. & TV Bands Database & $\begin{array}{l}\text { This is a service that TVBDs } \\
\text { must contact to submit their } \\
\text { operational location and obtain } \\
\text { TV channels availability. }\end{array}$ \\
\hline 4. & Rule & $\begin{array}{l}\text { A relationship between clauses } \\
\text { and depending on the situation } \\
\text { can be used to generate new } \\
\text { information or prove the truth } \\
\text { of an assertion }\end{array}$ \\
\hline 5. & Knowledge Base & $\begin{array}{l}\text { A set of if-then rules and } \\
\text { known facts }\end{array}$ \\
\hline 6. & Clause & Assertions or facts \\
\hline 7. & Inference Engine & $\begin{array}{l}\text { A collection of reasoning logic } \\
\text { used to process the rules and } \\
\text { data. }\end{array}$ \\
\hline 8. & Scanner & $\begin{array}{l}\text { A custom made object with the } \\
\text { capability of sensing free and } \\
\text { unoccupied channels and } \\
\text { assigning same to secondary } \\
\text { users. }\end{array}$ \\
\hline 9. & Cognitive Radio & $\begin{array}{l}\text { an intelligent wireless } \\
\text { communication system that is } \\
\text { aware of its surrounding } \\
\text { environment }\end{array}$ \\
\hline 10. & Genetic Algorithm & $\begin{array}{l}\text { Uses the principle of selection } \\
\text { and mutation to select best fit } \\
\text { individuals from a population } \\
\text { sample in a search space }\end{array}$ \\
\hline 11. & Chromosome & Blueprint for an individual \\
\hline 12. & Population & A collection of individuals \\
\hline 13. & Individual & $\begin{array}{l}\text { Any possible solution. In our } \\
\text { case any possible free and } \\
\text { unoccupied channel. }\end{array}$ \\
\hline 14. & Search Space & $\begin{array}{l}\text { All possible solutions to the } \\
\text { problem. In our case, all } \\
\text { available frequency bands in a } \\
\text { geographical location. }\end{array}$ \\
\hline 15. & Trait & Possible aspect of an individual \\
\hline 16. & Allele & Possible setting for a trait \\
\hline
\end{tabular}

\begin{tabular}{|l|l|l|}
\hline S/N & Object & Description \& Responsibility \\
\hline 17. & Locus & $\begin{array}{l}\text { The position of a gene in a } \\
\text { chromosome }\end{array}$ \\
\hline 18. & Genome & $\begin{array}{l}\text { Collection of chromosomes for } \\
\text { an individual }\end{array}$ \\
\hline 19. & Simulator & $\begin{array}{l}\text { An object that simulates signal } \\
\text { sensing and allocation to } \\
\text { secondary Users. }\end{array}$ \\
\hline 20. & Optimizer & $\begin{array}{l}\text { This object, optimizes the } \\
\text { allocation of free unoccupied } \\
\text { channels to devices with aid of } \\
\text { Inference Engine and Cognitive } \\
\text { Radio }\end{array}$ \\
\hline
\end{tabular}

\section{RESULT}

From the analysis made for the availability of TV white spaces in [3], the result shows that abundant of TV white spaces exist in Nigeria especially with ongoing digital switch over process but lacks proper algorithm and mechanism for their efficient allocation to WSDs. Considering the above our work has been able to overcome the allocation problem, equation 1 to 8 clearly shows that available channels that meets the criteria of the inference rules will be allocated and the geolocation database will at real time basis provide information about the channel status. Our model also took care of interference situation with the primary users by not allocation adjacent channels.

\section{CONCLUSION}

The TV white spaces have become an alternative means of salvaging the reoccurring decimal of spectrum scarcity. Ability to discover the existence of the TV white space availability is one issue and allocating them to the needing white space devices is another more serious issue. In this paper, we presented the design of hybrid model of fuzzy rule based technique and genetic algorithm. The model shows an optimal solution for the allocation of TV white spaces to requesting devices there by reducing it underutilization which in turn will free most portion of the spectrum.

\section{REFERENCES}

[1] Staple, G. and Werbach, K. (2011). The End of Spectrum Scarcity. IEEE Spectrum." IEEE Spectrum: Technology, Engineering, and Science News. Web. 23 Dec. 2011. http://spectrum.ieee.org/telecom/wireless/the-end-ofspectrum-scarcity

[2] Maheshwari, A. Gopalakrishnan, A. Harini, A. Mangla, N. Bhagavatula, P. Richa Goyat, R. (2012). Television White Spaces - Global Developments And Regulatory Issues In India

[3] Adigwe, W. Okonkwo, O. R (2017). Modeling TV White Spaces Availability Prediction for White Space Devices Utilization In Delta North Zone of Delta State Nigeria. Published in American Scientific Research Journal for Engineering, Technology, and Sciences (ASRJETS) (2017) Volume 34, No 1, pp 160-173

[4] Gawde, V. Mishra, B. K. \& Bansode, R. (2015). Global Journal of Computer Science and Technology: Network, 
Web \& Security Volume 15 Issue 8 Version 1.0 Year 2015 Pp 2-3.

[5] Nishant, R. P. (2014). Channel Allocation in Wireless Communication using Genetic Algorithm International Journal of Engineering and Innovative Technology (IJEIT) Volume 4, Issue 5, November 2014 Pp 6

[6] Clemens, N. and Rose, C. (2102). Intelligent Power Allocation Strategies in an Unlicensed Spectrum WINLAB at Rutgers University, Piscataway, New Jersey 08854

[7] Jayaraj, V. Jegathesh, J. A. Hemalatha, S. (2014). An Analysis of Genetic Algorithm and Tabu Search Algorithm for Channel Optimization in Cognitive AdHoc Networks. International Journal of Computer Science and Mobile Computing Vol. 3, Issue. 7, July 2014, Pp 60 $-62$
[8] Maninder, J. K. Moin, U. Harsh K. V. (2012). Performance Evaluation of CSMA/TDMA Cognitive Radio Using Genetic Algorithm, International Journal of Soft Computing and Engineering (IJSCE) Volume 2, Issue-3, July 2012.

[9] Yongcheng, L. Hai, S. Manxi, W. (2016). Spectrum Allocation of Cognitive Radio Based on Autonomy Evolutionary Algorithm, Cybernetics and Information Technologies Volume 16, No 4.

[10] Ogude U. C. and Onyejegbu L N. (2015). An Optimized Service Selection System Using Fuzzy Logic and Genetic Algorithm. African Journal of Computing \& ICT, Vol 8. No. 3 - December, 2015.

[11] Haupt, R. L and Haupt, S. E. (2004). Practical Genetic Algorithms Second edition Published by John Wiley \& Sons, Inc., Hoboken, New Jersey pp 150-170. 